\title{
Hernie De Amyand Compliquee De Gangrene De Fournier : A Propos D'un Cas Et Revue De La Litterature
}

\author{
Raouf Fayisall Geraldo, \\ Service de Chirurgie Viscérale et digestive, \\ Centre Hospitalier Moulins-Yzeure, France \\ Hugues Ndasu Matendo, \\ Liviu Musteata, \\ Valériu Krasovski, \\ Valentin Nitu, \\ Thierry Gastaud, \\ Fabrice Cattan,
}

Faculté des Sciences de la Santé, Université de Lomé, Togo

Service de Chirurgie Viscérale et digestive,

Centre Hospitalier Moulins-Yzeure, France

Doi:10.19044/esj.2020.v16n18p209 URL:http://dx.doi.org/10.19044/esj.2020.v16n18p209

\section{Résumé}

La hernie de Amyand se définit par l'incarcération de l'appendice vermiculaire dans le sac herniaire. Elle constitue une entité rare. Nous rapportons le cas d'un patient de 75ans, éthylique, dénutri et porteur d'une cardiopathie ischémique stenté et sous traitement anticoagulant, admis pour une tuméfaction douloureuse et chaude localisée en région inguinale droite évoluant depuis cinq jours. Le bilan biologique révélait un syndrome infectieux et des troubles sévères de l'hémostase. Le scanner a objectivé un appendice perforé au sein du sac herniaire droit et un thrombus intraventriculaire gauche. L'évolution s'est faite vers une gangrène de Fournier. Après correction des troubles hémostatiques, la prise en charge chirurgicale a consisté en l'appendicectomie, drainage par laparotomie médiane associée à une orchidectomie droite sur un testicule nécrosé avec exérèse jusqu'en zone saine de la peau scrotale. Malgré des constantes stables en per-opératoire, le patient a présenté un arrêt cardiaque en post-opératoire immédiat, réanimé sans succès.

Mots-clés: Hernie Amyand, Gangrène De Fournier, Thrombus Ventriculaire, Dénutrition, Traitement 


\title{
Amyand Hernia Causing a Fournier's Gangrene: A Case Report and Review of the Litterature
}

\author{
Raouf Fayisall Geraldo, \\ Service de Chirurgie Viscérale et digestive, \\ Centre Hospitalier Moulins-Yzeure, France \\ Faculté des Sciences de la Santé, Université de Lomé, Togo \\ Hugues Ndasu Matendo, \\ Liviu Musteata, \\ Valériu Krasovski, \\ Valentin Nitu, \\ Thierry Gastaud, \\ Fabrice Cattan, \\ Service de Chirurgie Viscérale et digestive, \\ Centre Hospitalier Moulins-Yzeure, France
}

\begin{abstract}
Amyand hernia is defined by the presence of appendix herniating into the deep inguinal ring. It's a rare condition. We report the case of a 75-yearold male patient, presenting alcoholism, undernutrition and ischemic cardiopathy which required angioplasty and anticoagulation, admitted for a painful and hot swelling in right inguinal region evolving for five days. Blood analysis revealed infectious syndrome and important hemostasis disorders. Computed Tomography scan showed perforated appendix herniating through inguinal ring associated to a thrombus in the left ventricle. The evolution was marked by a Fournier's gangrene. After getting the normalization of the hemostasis values, a laparotomy was performed for appendicectomy, drainage. The right testicle, necrotized, was removed, with a wide debridement of the fasciitis. Although the parameters were stable during the surgery, the patient presented a heart failure with unsuccessful cardiopulmonary resuscitation.
\end{abstract}

Keywords: Amyand Hernia, Fournier's Gangrene, Ventricular Thrombus, Undernutrition, Treatment 


\section{Introduction}

La hernie de Claudius Amyand se définit par la présence de l'appendice au sein d'un sac herniaire. C'est une entité rare (Rajaguru et Tan, 2016). Son évolution peut se faire vers un abcès, une péritonite généralisée. De même, elle expose à une diffusion vers le scrotum par voie de contiguïté, point de départ d'une gangrène périnéale survenant plus ou moins rapidement selon l'état du patient. Le pronostic vital est alors engagé du fait du sepsis. Nous rapportons ici un cas de hernie de Amyand compliquée de gangrène de Fournier chez un patient atteint de cardiopathie. Les objectifs sont de décrire les circonstances du diagnostic, la prise en charge et son évolution puis de faire une revue de la littérature.

\section{Observation}

Un patient de 75 ans, a été admis aux urgences pour douleur de la région inguinale droite évoluant depuis cinq jours, associée à une fièvre et des nausées. Il n'y avait pas d'arrêt de matières ni de gaz. On ne notait pas de signes urinaires associés. Le patient était éthylique chronique (environ six verres / jour). Il était sous traitement anticoagulant (Fluindione comprimés $20 \mathrm{mg} /$ jour) suite à une angioplastie pour infarctus du myocarde. Cliniquement, le patient présentait une tuméfaction douloureuse, chaude en région inguinale droite, une fièvre à $38,3^{\circ} \mathrm{C}$, une tachycardie $(108$ battements par minute), une tension artérielle de $110 / 70 \mathrm{mmHg}$. L'examen des bourses était normal.

L'hémogramme a révélé une hyperleucocytose (16600 éléments $\left./ \mathrm{mm}^{3}\right)$ avec prédominance de polynucléaires neutrophiles sans anémie. La Protéine C Réactive à $335 \mathrm{mg} / \mathrm{L}$, le Taux de Prothrombine à 7\% et l'INR à 9,9. L'albuminémie était de $16 \mathrm{~g} / \mathrm{L}$. L'ionogramme sanguin révélait une hyperkaliémie rapidement normalisée. La fonction rénale et la glycémie étaient normales. Le scanner thoraco-abdominal a révélé une hernie inguinoscrotale avec contenu digestif sous forme d'une appendicite perforée et un thrombus intra ventriculaire gauche (Figure 1). 


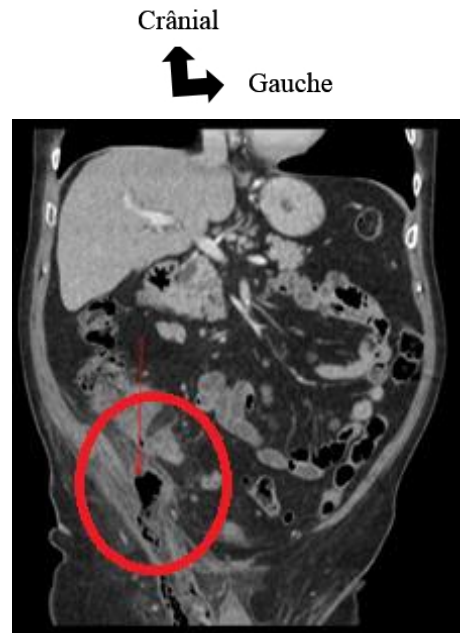

$1 \mathrm{~A}$

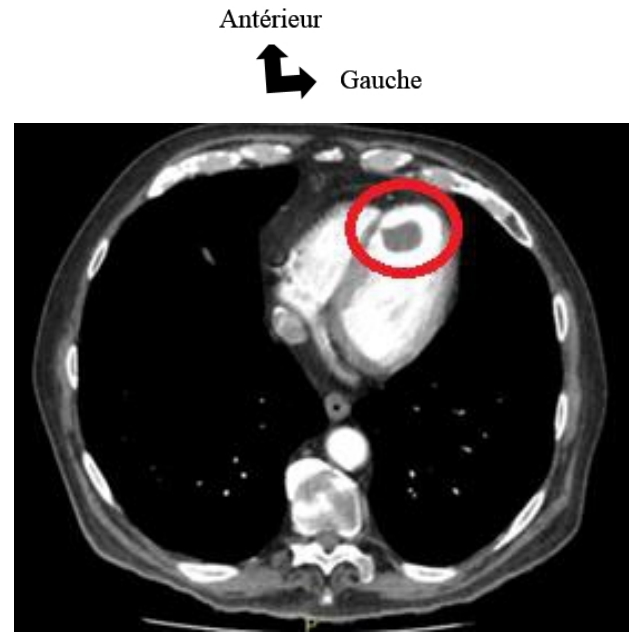

1B

Figure 1 (1A et 1B) : Scanner abdominal injecté : 1A (reconstruction frontale montrant une hernie inguino-scrotale droite avec contenu digestif sous forme d'une appendicite perforée) et $1 \mathrm{~B}$ (coupe thoracique basse montrant un thrombus dans le ventricule gauche)

Une vitaminothérapie a été instaurée en urgence (Vitamine K1 injectable $2 \mathrm{mg} / 24$ heures), associée à une triple antibiothérapie (Ceftriaxone injectable $2 \mathrm{~g} / 24$ heures, Métronidazole injectable 500mg / 8 heures, Gentamycine injectable 160mg / 24 heures), avec amélioration du bilan d'hémostase (TP à $46 \%$ et INR à 1,9) après 24 heures de traitement. L'échographie cardiaque trans-thoracique a confirmé une dysfonction ventriculaire gauche sévère avec une akinésie apicale, une fraction d'éjection estimée entre 25 et $30 \%$, et l'aspect d'un thrombus à l'apex plutôt mobile de $2.65 \mathrm{~cm} \times 1.85 \mathrm{~cm}$. L'avis de chirurgie cardiaque a été demandé qui a conclu à l'absence d'indication de prise de charge cardiaque dans ce contexte septique. L'évolution clinique fut marquée par l'apparition d'une infiltration des bourses avec une crépitation de la région périnéale et une plage de nécrose au niveau de la bourse qui a fait évoquer une gangrène de Fournier classant la hernie de Amyand type C de Fernando et Leelaratna ; type III de Losanoff et Basson avec décision opératoire en urgence.

Une laparotomie fut indiquée sous anesthésie générale avec une voie veineuse centrale et un cathéter intra-artériel. Par un double abord abdominal et scrotal, il a été découvert une masse inflammatoire réalisant un abcès sur appendice gangréné à base saine, fistulisée dans le canal inguinal et la bourse droite (Figure 2). Il a été réalisé une appendicectomie avec décision de cure herniaire différée. Puis un abord scrotal droit a été réalisé avec orchidectomie droite sur un testicule nécrosé, une large exérèse jusqu'en zone saine de la peau scrotale. Un double drainage par voie abdominale et par voie scrotale a été réalisé. 

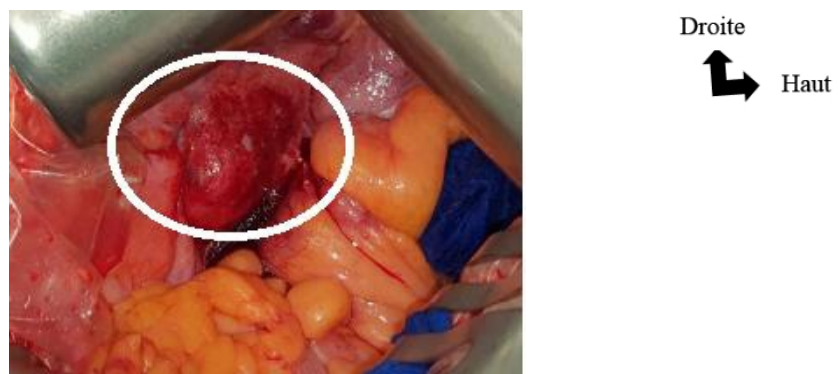

Figure 2 : Image peropératoire montrant une masse inflammatoire en fosse iliaque droite

Le patient, dont les paramètres hémodynamiques étaient stables lors de l'intervention a présenté un arrêt cardiaque en salle de réveil. La réanimation cardio-pulmonaire fut réalisée pendant près de 30 minutes sans succès.

\section{Discussion}

La hernie de Claudius Amyand se définit par la présence au sein du sac herniaire de l'appendice normal, inflammatoire ou perforé (Sharma et al., 2007). C'est une entité rare, sa fréquence globale est de $0,1 \%$ (Rajaguru et al., 2016). Elle représente $1 \%$ des hernies étranglées contenant des viscères et 0,13\% des formes d'appendicites (Ryan, 1937). Elle se distingue de la hernie de Littré (contenant un diverticule de Meckel), de la hernie de Richter (contenant le bord anti-mésentérique des anses intestinales) et de la hernie de De Garengeot (appendice au sein d'une hernie fémorale (Talini et al., 2015). La hernie de Amyand a également été décrite à gauche en rapport avec des cas de malrotation intestinale et de situs inversus (Ghafouri et al., 2012).

Dans la littérature, la hernie de Amyand survient chez les sujets adultes jeunes. La moyenne d'âge est de 42 ans, bien que des cas ont été décrits chez les enfants (Sharma et al., 2007). La survenue de l'inflammation de l'appendice au sein du sac herniaire s'explique par deux mécanismes: obstruction endoluminale par des obstacles tels que les stercolithes ou compression extrinsèque par l'orifice musculo-pectinéal de Fruchaud Hasselbach (Singal et al., 2012).

Son diagnostic se base sur l'association de signes. Cliniquement, elle se présente sous la forme d'une hernie étranglée avec ou sans signes d'occlusion intestinale (Ghafouri et al., 2012). Sur le plan biologique, il existe un syndrome inflammatoire. Le scanner abdominal permet d'orienter vers le diagnostic même si dans la majorité des cas, le diagnostic est peropératoire (Luchs et al., 2000).

Son traitement répond aux principes de prise en charge d'une hernie étranglée. Il comprend un temps viscéral consistant en l'appendicectomie 
suivie d'une réparation pariétale immédiate ou différée ; généralement par raphie en raison du risque infectieux sur matériel prothétique.

La prise en charge tardive ou inappropriée entraîne des complications, avec une importante morbi-mortalité. En effet, le décès survient chez trois patients sur 10 (Carey, 1967). L'extension des processus infectieux intraabdominaux vers le périnée entraînant une gangrène de Fournier est peu décrite. En 1991, la première description d'un Fournier dû à une appendicite a été faite chez un patient de 27 ans. Il s'agissait d'une appendicite gangréneuse dont l'extension s'est faite via le conduit déférent. La prise en charge a consisté en une antibiothérapie associée à l'appendicectomie avec nécrosectomie et drainage. Il n'y avait pas de hernie associée. Les suites ont été simples (Gaeta et al., 1991). Par la suite, cinq autres cas similaires ont été rapportés (Gerber et al., 1994 ; Ubieto et al., 2002 ; Osawa et al., 2005). En 1994, Kikuchi et al. ont décrit un cas de Syndrome de Fournier dans les suites d'une appendicectomie chez un enfant de 10ans.

Toutefois, dans la littérature, seulement quatre cas de gangrène de Fournier en rapport avec une hernie de Amyand ont été répertoriés.

Sarmah et al. en 2015 ont rapporté le cas d'un patient de 68ans, non diabétique sous anticoagulants (Warfarine) pour thrombus intraventriculaire gauche, qui a présenté une douleur en fosse iliaque droite évoluant depuis trois jours. Il présentait des signes de choc septique, une nécrose périnéale à point de départ la fosse iliaque droite. Le scanner a montré la présence de gaz dans l'hémiscrotum droit. La correction des troubles hémostatiques a consisté en l'administration de concentré de Prothrombine. Le premier temps opératoire a consisté en une exérèse large des tissus nécrosés en région périnéale avec orchidectomie droite. Devant la découverte de l'écoulement purulent provenant du canal inguinal, une laparotomie a été ensuite réalisée. Un appendice inflammatoire a été retrouvé, avec caecum ischémique. Une hémicolectomie droite a été réalisée. Le patient a présenté un arrêt cardiorespiratoire en post-opératoire immédiat avec mesures de réanimation sans succès.

En 2016, Rajaguru et al. ont rapporté le cas d'un patient de 47ans, présentant comme comorbidités une hyperthyroïdie, des troubles mentaux et une hypertension artérielle. Il a présenté le même tableau clinique avec quatre jours d'évolution. Le traitement chirurgical a consisté en une appendicectomie première suivie d'une détersion périnéale. La réparation pariétale a été réalisée après 48 heures en raison du risque infectieux et l'état nutritionnel. Elle a consisté en une cure herniorraphie selon la technique de Bassini. Les suites ont été favorables.

En 2019, un troisième cas a été décrit chez un patient immunocompétent de 35ans, sans antécédents connus admis pour une appendicite aiguë évoluant depuis 48 heures et ayant conduit à une gangrène 
de Fournier (Alzerwi et al., 2019). La prise en charge a été chirurgicale avec appendicectomie et détersion mécanique. Les suites opératoires étaient favorables.

Sladek et al. ont rapporté un cas similaire en 2019 chez un patient de 70ans.

L'orchidectomie est donc souvent réalisée de façon concomitante. Toutefois, le testicule est le plus souvent sain bien que les tissus avoisinants soient nécrosés (Yanar et al., 2006). Concernant la réparation pariétale, les techniques de raphie sont adoptées. Toutefois, il n'y a pas de consensus sur le délai de ce temps pariétal (immédiat ou différé).

L'évolution a été rapide chez le patient de notre observation, due aux facteurs d'immunodépression et de dénutrition sévère qu'il présentait; associé à un éthylisme chronique. Pa ailleurs, le délai d'admission était long (cinq jours d'évolution). Les comorbidités du patient et l'absence initiale des signes de gangrène expliquent la prise en urgence différée avec une chirurgie écourtée. En effet, un délai de 24 heures fut nécessaire en vue de la correction des troubles de l'hémostase ainsi que le conditionnement anesthésique du fait du thrombus mobile intra-ventriculaire gauche. Le décès peut être dû à un infarctus massif du myocarde, dans un contexte septique.

\section{Conclusion}

La hernie de Amyand est rare. Selon l'état du patient, l'évolution peut être fulgurante vers une atteinte périnéale. La gangrène de fournier qui en résulte engage le pronostic vital. Elle est peu rapportée dans la littérature. Le diagnostic est souvent fait en per-opératoire. Le traitement est médical (antibiotiques) et chirurgical qui doit être précoce et agressif. L'appendicectomie est systématique, associée à une réparation pariétale par raphie immédiate ou différée avec excision large des tissus dévitalisés. En cas de gangrène de Fournier, le pronostic est généralement réservé.

\section{Conflit d'intérêts}

Les auteurs déclarent ne pas avoir de liens d'intérêt.

\section{References:}

1. Alzerwi NA, Alshanwani M, Alsultan AS, Almutairi S, Aldebasi YI, Ali BI. Perforated appendicitis as a source of Fournier's gangrene in an immunocompetent male. Int Surg J 2019; 6(10):3813-6.

2. Carey LC. Acute appendicitis occurring in hernias: A report of 10 cases. Surgery 1967; 61:236-8.

3. Carolina Talini, Luan Ocaña Oliveira, Allan César Faria Araújo, et al. De Garengeot hernia: Case report and review Int J Surg Case Rep. 2015; 8: 35-7. 
4. Gaeta M, Volta S, Minutobi A, Bartiromo G, Pandolfo I. Fournier gangrene caused by a perforated retroperitoneal appendicitis: CT demonstration. AJR 1991;156(2):341-2.

5. Gerber GS, Guss SP, Pielet RW. Fournier's gangrene secondary to intra-abdominal processes. Urol 1994; 44(5):779-82.

6. Ghafouri A, Anbara T, Foroutankia R. A rare case report of appendix and cecum in the sac of left inguinal hernia (left Amyand's hernia). Med J Islam Repub Iran 2012; 26(2):94-5.

7. Kikuchi N, Shibata M, Komura Y, Yokota S, Mayumi T, Sugimoto H, et al. Fournier's syndrome in a 10-year-old boy after appendicectomy. 1994 ;5(4):395-8.

8. Luchs JS, Halpern D, Katz DS. Amyand's hernia: Prospective CT diagnosis. J Comput Assist Tomogr 2000; 24:884-6.

9. Osawa H, Takahashi H, Sakata I. A case of Fournier gangrene caused by perforated acute appendicitis. 2005; 25(7):941-5.

10. Piyush B. Sarmah, Mashuk Khan, Miguel Zilvetti. Fournier's gangrene secondary to an acutely inflamed appendix herniating into the deep inguinal ring. Journal of Surgical Case Reports, 2015, 1-3.

11. Rajaguru K, Tan Ee Lee D. Amyand's hernia with appendicitis masquerading as Fournier's gangrene: a case report and review of the literature. J Med Case Reports 2016; 10:263.

12. Ryan WJ. Hernia of the vermiform appendix. Ann Surg 1937; 106:135-9.

13. Sharma H, Gupta A, Shekhawat NS, et al. Amyand's hernia: a report of 18 consecutive patients over a 15-year period. Hernia 2007; 11(1):31-5.

14. Singal R, Mittal A, Gupta A, et al. An incarcerated appendix: report of three cases and a review of the literature. Hernia. 2012; 16(1):91.

15. Sladek F, Krass V, Salas O, Sutory M, Pasa L. Fournierova gangréna jako komplikace Amyandovy hernie. Rozhl Chir 2019; 98(7):291-6.

16. Ubieto MF, Coello RJ, Murillo SJ, Andreu AM, Bienzobas RJ, Salinas PV, et al. Testicular necrosis caused by Fournier's gangrene: an exceptional case. Arch Esp Urol 2002;55(3):309-11.

17. Yanar H, Taviloglu K, Ertekin C, et al. Fournier's gangrene: risk factors and strategies for management. World Journal of Surgery 2006; 30(9):1750-4. 